\title{
CUATRO DÉCADAS DE LA REVISTA PARADIGMA. Producción, Autoría, Filiación Institucional y Distribución Geográfica
}

\author{
Ma. Margarita Villegas \\ Universidade Federal Rural do Semi Árido (UFERSA; Mossoró, Brasil) \\ margaritavillega@hotmail.com \\ http://orcid.org/0000-0002-4965-2291 \\ Fredy Enrique González \\ Universidade Federal do Rio Grande do Norte (UFRN, Brasil) \\ fredygonzalezdem@gmail.com \\ http://orcid.org/0000-0002-8079-3826 \\ Herminia Vincentelli \\ https://orcid.org/0000-0002-5224-2023 \\ herminiavincentelli@hotmail.com \\ Universidad Pedagógica Experimental Libertador (UPEL, Venezuela)
}

Recibido: 04/04/2020 Aprobado: 10/06/2020

\section{Resumen}

En este artículo, además de una breve síntesis cuantitativa de los artículos publicados en Paradigma desde su primera edición (julio de 1980) hasta la actualidad (junio de 2020), se realiza un análisis del contenido de la edición con la cual esta publicación conmemora su cuadragésimo aniversario, en función de los siguientes parámetros: autoría, filiación institucional y distribución geográfica de los 42 artículos que conforman dicha edición. El estudio se sustenta teóricamente en las nociones propias de la comunicación científica y la apreciación de la calidad de las publicaciones periódicas académicas. Metodológicamente, se trata de un estudio documental que usó como corpus el Índice Acumulado (1980-2020) de la revista y los resúmenes de los 42 artículos. Para el examen de la información cuantitativa se aplicaron técnicas simples de contaje de frecuencia, mientras que la información cualitativa fue obtenida a través de la lectura del resumen de cada trabajo. De los autores se destaca, la predominancia del género femenino $(62,35 \%)$; la pertenencia a diferentes áreas disciplinarias, así como su afiliación a 45 instituciones, de las cuales 43 son universidades, ubicadas en 12 países (2 europeos, 1 asiático, 8 latinoamericanos y EEUU). Todo ello, resalta la visibilidad de Paradigma; evidenciándose así, la importancia de las revistas como medio para el estudio y justificación de la inversión en ciencia que se hace en las instituciones de educación superior y, para este caso, las vinculadas con la formación de profesores.

Palabras Clave. Producción Científica. Comunicación Científica. Revisión por Pares. 


\title{
QUATRO DÉCADAS DA REVISTA PARADIGMA. Produção, Autoria, Filiação Institucional e Distribuição Geográfica
}

\begin{abstract}
Resumo
Neste artigo, além de uma breve síntese quantitativa dos artigos publicados no Paradigma desde sua primeira edição (julho de 1980) até os dias atuais (junho de 2000), é feita uma análise do conteúdo da edição com a qual essa publicação comemora seu quadragésimo aniversário, com base nos seguintes parâmetros: autoria, afiliação institucional e distribuição geográfica dos 42 artigos que compõem esta edição. Teoricamente, o estudo se baseia nas noções de comunicação científica e na apreciação da qualidade dos periódicos acadêmicos. Metodologicamente, tratase de um estudo documental que utilizou como corpus o Índice Acumulado (1980-2020) da revista e os resumos dos 42 artigos. Para o exame das informações quantitativas, foram aplicadas técnicas simples de contagem de frequências, enquanto as informações qualitativas foram obtidas através da leitura do resumo de cada trabalho. Dos autores, destaca-se a predominância do gênero feminino (62,35\%); pertencentes a diferentes áreas disciplinares e afiliação a 45 instituições, das quais 43 são universidades, localizadas em 12 países ( 2 europeus, 1 asiático, 8 latino-americanos e EUA). Tudo isso destaca a visibilidade da Revista Paradigma; evidenciando, assim, a importância dos periódicos como meio de estudar e justificar o investimento em ciências realizado nas instituições de ensino superior e, nesse caso, aquelas relacionadas à formação de professores.
\end{abstract}

Palavras chave. Produção científica. Comunicação científica. Revisão por pares.

\section{FOUR DECADES FROM THE PARADIGM MAGAZINE. Production, Authorship, Institutional Filiation and Geographical Distribution}

\begin{abstract}
In this article, in addition to a brief quantitative synthesis of the articles published in Paradigma from its first edition (July 1980) to the present day (June 2000), an analysis is made of the content of the edition with which this publication celebrates its publication for his fortieth anniversary, based on the following parameters: authorship, institutional affiliation and geographical distribution of the 42 articles that make up this edition. Theoretically, the study is based on the notions of scientific communication and the assessment of the quality of academic journals. Methodologically, this is a documentary study that used the Paradigma's Accumulated Index (1980-2020) and the abstracts of the 42 articles as corpus. For the examination of quantitative information, simple techniques for counting frequencies were applied, while qualitative information was obtained by reading the summary of each work. The authors, there is a predominance of females $(62,35 \%)$; belonging to different disciplinary areas and affiliation to 45 institutions, of which 43 are universities, located in 12 countries (2 European, 1 Asian, 8 Latin American and USA). All of this highlights the Revista Paradigma's visibility; thus evidencing the importance of journals as a means of studying and justifying the investment in science that is made in higher education institutions and, in this case, those related to teacher education.
\end{abstract}

Key words. Scientific production. Scientific communication. Peer review. 


\section{Introducción}

La revista Paradigma es una publicación científica, periódica cuyo ámbito es la formación, inicial y permanente de profesores de diferentes especialidades, quienes actúan tanto en instituciones de educación superior como de otros niveles y modalidades de los sistemas educativos de los países que representan; puede ser valorada como una publicación científica, ya que, de acuerdo con lo expuesto por Goncalves, Costa y Figueredo (2006), constituye un vehículo de comunicación formal y está organizada en fascículos o números que son publicados según una periodicidad definida, con tiraje y distribución amplia, cuyos contenidos son predominantemente artículos científicos.

Existe consenso, tal como lo expresan Bufrem; Gabriel Junior y Gonçalves (2010) en que es necesario dar a conocer la producción de los(as) investigadores(as), por cuanto esta acción, además de ser un compromiso social, es vital para la existencia de la propia ciencia. En relación con este aspecto, González (2015), al identificar las macro etapas de un trabajo de investigación, indica que una de ellas es la etapa de la comunicación, la cual implica

[...] la redacción del informe escrito, la elaboración de recursos para su exposición y defensa pública y la producción de al menos un artículo asociado con el trabajo que ponga a disposición de la comunidad interesada los pormenores del estudio realizado. Todas estas macro etapas son cruciales, especialmente la de Comunicación pues es ésta la que viabiliza la transferencia y aplicación de los logros alcanzados por la investigación, con lo cual ésta se legitima y refuerza su valor social. (González, 2015, p. 282). (cursivas añadidas)

Esta idea ya había sido expuesta por Campos (2000) al afirmar que un experimento científico no termina mientras no hayan sido publicados sus resultados; por lo tanto, hacer ciencia implica también escribir ciencia.

Puede entonces afirmarse que la comunicación científica (Pasquali, 1990) es el "contexto de justificación" (Bárcenas, 2002) de las revistas, como el caso de Paradigma, pues ellas constituyen un medio que contribuye a viabilizar la divulgación, difusión y diseminación así como también la discusión y validación de los resultados de las investigaciones, lo cual es esencial para el desarrollo idóneo de la ciencia. Por su parte, Cetto (citada por Haupt, 2000) estima que la creación de las revistas científicas obedece a una serie de motivos subyacentes, entre los cuales destaca el deseo de los autores de contar con un medio que permita dar una 
mayor visibilidad a su producción académica y científica. Además de este interés individual, prevalece un interés social.

En efecto, las revistas son, al mismo tiempo, promotoras e indicadoras de la actividad científica, al constituirse como medios de auto, co y hetero evaluación; esto se logra mediante el proceso de revisión por pares (o peer review ${ }^{l}$ ) que, de acuerdo con Kelly, Sadeghieh, y Adeli (2014), consiste en "someter el trabajo académico, la investigación o las ideas de un autor al escrutinio de otros expertos en el mismo campo" (p. 228). Estos mismos autores agregan que:

La revisión por pares está destinada a servir a dos propósitos principales. En primer lugar, actúa como un filtro para garantizar que solo se publique una investigación de alta calidad, especialmente en revistas acreditadas, al determinar la validez, importancia y originalidad del estudio. En segundo lugar, la revisión por pares está destinada a mejorar la calidad de los manuscritos que se consideran adecuados para su publicación. Los revisores (árbitros) brindan sugerencias a los autores sobre cómo mejorar la calidad de sus manuscritos y también identifican los errores que deben corregirse antes de la publicación. (p. 228) (paréntesis añadidos y traducción libre)

Se observa que la intencionalidad de la revisión por pares es garantizar e incrementar la calidad de lo que aparezca publicado en las revistas científicas, debido a que, como lo destaca Luchillo (2019), ellas "son el soporte principal de la difusión de los resultados de la investigación y, al mismo tiempo, constituyen la pieza clave del reconocimiento científico para la carrera de los investigadores y el prestigio de las instituciones" (p. 44).

Esta clase de publicaciones se basa en el principio de la validación del mérito por la comunidad científica (Ramírez, 2009), mediante la cual se exponen a la consideración de los pares los logros alcanzados por la comunidad de investigadores(as) de una determinada área de conocimiento; quienes, al hacerlo público, cumplen con el fin último de la ciencia, que es colocar los hallazgos científicos al servicio de los individuos que conforman la sociedad (Goncalves, Costa y Figueredo, 2006). Por ello, en palabras de Ramírez (2009) “[...] la investigación tiene que ser divulgada, proceso encargado a publicaciones periódicas especializadas, calificadas, certificadas y reconocidas para que puedan ser aceptadas, verificadas y reproducidas por la comunidad científica [...]" (p. 218).

\footnotetext{
1 “What Is Peer Review?” (2014). Int J Comput Appl. Disponible em: https://www.ijcaonline.org/what-is-peerreview Acceso: 09 de junio de 2020.
} 
En ese mismo sentido, Ramírez y Ramírez (2009) considera la divulgación del conocimiento, tanto formal (revistas especializadas, literatura gris y congresos científicos) como informalmente (e-mail, redes de colaboración), como un hecho fundamental para la comprensión de la organización social de la ciencia.

En cuanto a las funciones básicas de la comunicación científica, Paisley (1984) identifica las cuatro siguientes: (a) dar a conocer los hallazgos a la comunidad científica; (b) contribuir al progreso de las respectivas ciencias, (c) propiciar la discusión, la retroalimentación del científico con sus pares; y (d) recompensar al investigador mediante el reconocimiento de la comunidad científica a la que pertenece.

La importancia atribuida a las revistas como medio de comunicación científica, es un asunto de carácter histórico. En efecto, su emergencia, de acuerdo con Meadows (1999) y Burke (2003), ha sido ubicada en el año 1665 cuando en Paris fue fundado el "Journal des Scavans", cuyos propósitos iniciales fueron: catalogar los libros más importantes de Europa, publicar informes científicos y publicar noticias del mundo de las letras. Posteriormente, en marzo de ese mismo año, en Londres fue creada la revista Philosophical Transactions de la Royal Society of London (RSL), por Henry Oldenburg (c.1619-1677), primer secretario de la SRL y editor fundador de esta publicación que aún está activa. En ese entonces, publicaba artículos sobre nuevas ideas e investigaciones y cartas que se intercambiaban entre si los miembros de la comunidad científica.

En la sociedad actual, caracterizada por el desarrollo exponencial del conocimiento, producto de la importancia que se otorga a la creación de ciencia y tecnología como indicador de desarrollo de un país (Barrere, 2019), se ha generado una disposición favorable para la difusión de la ciencia que, de manera gradual, ha convertido la comunicación científica en una tarea ineludible para las instituciones académicas, asociaciones científicas y otros agentes vinculados con los procesos de producción de conocimiento. Entre los factores que destacan en ese incremento están "[...] el crecimiento acelerado del número de estudiantes de ciencia, de las comunidades científicas, de las demandas de las bibliotecas universitarias, y de la oferta creciente de trabajos de alta calidad científica [...]”' (Barsky, 2014, p 160),

Así, se reconoce que la calidad de las revistas científicas está en relación con la política de inversión, de los insumos otorgados para los científicos, del empeño de los gobiernos para 
aminorar la brecha, producto de los cambios y avances que en este ámbito se operan en el plano regional e internacional (Cetto, citada por Haupt, 2000).

Esto parece NO haber sido bien comprendido por los gobiernos de América Latina y el Caribe (ALC) por cuanto el porcentaje de su PIB asignado a inversión en investigación y desarrollo es escaso, tal como ha sido señalado por Requena (2003) y Vessuri (2006), con cifras que expresan dramáticas diferencias en relación con las naciones industrializadas; siendo lo anterior confirmado por estudios posteriores realizados en ALC durante el periodo de 2008 al 2017, reflejados en el informe coordinado por Barrere (2019), en donde se muestra que en la región, la inversión en $\mathrm{I}+\mathrm{D}$ continúa sin responder a las expectativas deseadas, ya que el bajo porcentaje del PIB destinado para dicha inversión, se ubica, en ese periodo, en 0,64\%, siendo este ínfimo si se lo compara con los países desarrollados, algunos de los cuales superan el 3 y otros el $4 \%$ (Alemania, USA, Corea del Sur); no obstante, en ALC (según Barrere, 2019), ha habido un significativo aumento en el número de investigadores en ese mismo periodo (2008 a 2017) de un $28 \%$, así como también se pudo constatar que ha habido un aumento de $84 \%$ en cantidad de artículos científicos publicados durante el mismo (p.12).

Es importante destacar que esas cifras muestran la creciente concientización de los gobiernos de ALC por los indicadores de progreso y desarrollo establecidos por organismos internacionales (Barsky, 2014; Luchillo, 2019), entre los cuales están incluidos los indicadores bibliométricos que constituyen la referencia por excelencia para determinar la productividad, visibilidad, impacto e influencia de la actividad científica de un país, región, comunidad científica, instituciones académicas, área de conocimiento, entre otras muchas (Barrere, 2019; López, Jiménez, Ruiz 2009).

En ese contexto, el análisis de la producción científica publicada en revistas académicas, de acuerdo con Luchillo (2019); Delgado, Jiménez, Ruiz, (2009); Witter (1999); Mourao, (1999), son una fuente para la validación de la producción científica de los investigadores, instituciones académicas y otras instancias dedicadas al quehacer investigativo a fin de valorar su impacto social y sus hallazgos, obtener una mejor explicación y comprensión de los fenómenos naturales o socioculturales que les condicionan y, de esa manera, evaluar los avances científicos de un país con respecto a otro. Es decir, estas ayudan a: la evaluación de los científicos y de la ciencia; la formación y renovación de cuadros de investigadores; la estructuración de proyectos de investigación; la consolidación de campos científicos; y, 
también, al incremento de la memoria científica, o exomemoria como la denominan los científicos de la información (García, 2001), mediante la creación de repositorios del conocimiento (Laborde, 2009, p. 714).

Sobre este particular, Ramos (2001) sostiene que la evaluación de las revistas académicas es un proceso documental necesario para la elaboración de índices lo que permite el acceso a los contenidos de las publicaciones y de esta manera se le da visibilidad al conocimiento. De esos estudios se derivan conclusiones y orientaciones en relación con la consolidación de la publicación, impacto del contenido de la revista, justificación de la inversión para la investigación, toma de decisiones para reorientar aspectos del proceso editorial que así lo requieran (Meza, 2005), ya que el desarrollo de instituciones se sostiene gracias a la investigación de quienes ejercen docencia en las universidades (Tapia (2006).

Refiriéndose a este mismo asunto, Witter (1996) destaca la importancia que tiene para los lectores de ciencia, el análisis sistemático de los trabajos científicos publicados en los distintos medios disponibles, ya que propicia una visión general sobre los intereses de los investigadores en un determinado período histórico, además de apuntar las estrategias empleadas por los estudiosos para la selección, tratamiento e interpretación de la información con miras a construir sus datos de pesquisa.

Ubicándonos en el ámbito venezolano, Ruiz (1991, citado por Vincentelli y Witter, 2009) afirma que la preocupación por la comunicación científica en el área de educación se remonta al siglo XIX. Dicho autor señala que en 1871 se crean las revista Abecé, El Instructor Venezolano (1891) y la Revista de Instrucción Pública (1893). Varias décadas después, se funda la Revista de Educación (1936) y Educación (1939), creadas, todas ellas, por iniciativa del organismo rector de la educación, -en ese entonces llamado Ministerio de Instrucción Públicacon el propósito de informar y orientar a los maestros sobre diversos aspectos relacionados con el quehacer docente.

Cabe destacar que estas publicaciones, debido a la presencia de múltiples factores, entre ellos, la falta de continuidad en las políticas académico-administrativas, escasa proyección institucional de las respectivas publicaciones, dificultades derivadas de la poca participación de los docentes en la publicación de contenidos, condicionaron la corta existencia de las publicaciones antes mencionados, a excepción de la revista "Educación" que tuvo una larga trayectoria. 
Tal como lo indican Vincentelli y Witter (2009), desde hace aproximadamente dos décadas, la situación ha mejorado de manera significativa, sin llegar a ser la ideal, hoy en día, tanto las universidades públicas como las privadas, desarrollan políticas editoriales que favorecen el mejoramiento de las publicaciones científicas y la permanencia de las mismas tanto en formato electrónico como impreso, aunque debido al incremento de los costos la cantidad de publicaciones electrónicas es cada vez mayor. Se extreman esfuerzos para cumplir con las exigencias y criterios establecidos por las distintas bases de datos, así lo confirman, los registros de publicaciones periódicas LATINDEX (2020), que contabiliza 536 revistas venezolanas, en

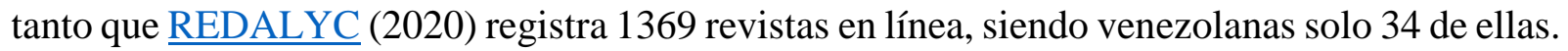
Otro repositorio importante es Scielo, cuya sección Venezuela registra 60 revistas de esta nación suramericana. En su gran mayoría, están adscritas a dependencias o centros de investigación de las respectivas universidades de origen. En el caso de las publicaciones periódicas centradas en el área de Humanidades, Scielo Venezuela registra 21 títulos, de los cuales 7 (un tercio) corresponden a Educación

El panorama que muestran estos tres repositorios era muy distinto al de 1980 cuando fue fundada Paradigma que, desde entonces ha persistido como medio de difusión del quehacer científico de profesionales de las ciencias de la educación, tanto venezolanos como de otros países latinoamericanos, europeos y norteamericanos quienes han contado con las páginas de esta revista para compartir su producción científica con sus pares y con las demás personas interesadas, por variadas razones, en asuntos propios de las diversas disciplinas educativas.

Cabe destacar que la producción de Paradigma ha sido asunto de interés indagatorio tanto para investigadores del área de Ciencias de la Información como también de otros ámbitos de la investigación social. Entre esos estudios puede mencionarse el realizado por Lara y Witter (1999) quienes estudiaron la producción científica de la revista Paradigma durante el lapso comprendido entre 1980 y 1997, considerando: título de los artículos (número de vocablos), género de los autores, tipo de autorías y temas estudiados. El análisis de los resultados indica, entre otros particulares, lo siguiente: el número de vocablos presentes en los títulos es superior a lo establecido por los patrones internacionales; predominio de autoría única, indicando rezago en la incorporación de las tendencias actuales del trabajo científico en equipos interdisciplinarios. También se constató mayor presencia de autoría masculina y en relación con los temas s tratados por los autores se observa que los contenidos más focalizados se refieren al 
área de enseñanza y aprendizaje y temas generales sobre educación. Fue significativa la dispersión de temas.

En esta misma línea, Vincentelli (2003) analizó la producción científica publicada en la revista Paradigma en el período 1990-1999. La autora evaluó las variables-categorías referentes a: temas estudiados, género de los autores, tipo de autoría y referencias bibliográficas. Se verificó que los temas más estudiados fueron teorías y metodologías de la investigación, planificación y administración educativa y enseñanza de disciplinas específicas. Con relación al género de los autores se registra mayor presencia de autores masculinos y prevalece autoría única.

Por su parte, González (2008), también revisó el contenido de lo publicado en la Revista Paradigma durante el lapso 1980 - 2007, considerando: (1) Género del Autor; (2) Tipo de Autoría; (3) Origen de la Contribución; (4) Instituciones Representadas; (5) Temática tratada. En este estudio se evidenció el predominio de la autoría masculina única, los artículos de origen nacional y una amplia representación de instituciones universitarias del país sede de la publicación.

Tomando en cuenta que, en Latinoamérica, según, Romero-Torres; Acosta-Moreno; Tejada-Gómez (2013), las revistas científicas tienen una vida relativamente corta, que Paradigma haya conseguido llegar a cuarenta años constituye una circunstancia relevante para la academia iberoamericana representada por sus autores. Y es esto lo que se desea destacar en el presente trabajo, cuyo propósito se expresa en los siguientes

\section{Objetivos}

1. Mostrar información cuantitativa sobre la producción científica de la Revista Paradigma, considerando los artículos publicados ella desde su primera edición (julio de 1980) hasta la actualidad (junio de 2020).

2. Analizar los artículos incluidos en la edición de Paradigma, correspondiente a su cuadragésimo aniversario, en función de los siguientes parámetros:
a. Autoría
b. Filiación institucional
c. Distribución Geográfica.
d. Temáticas Desarrolladas 


\section{Método}

Este estudio es de carácter documental; los materiales básicos de consulta fueron, por un lado, el Índice Acumulado de la Revista Paradigma, contentivo de información referida a todos los artículos publicados en la misma durante el período 1980-2020; y por otro, se tuvo acceso pleno a los artículos insertos en la edición del cuadragésimo aniversario, y de cada uno de ellos se analizó el correspondiente resumen, sin realizar alteración alguna en el mencionado índice ni en los resúmenes examinados.

En el tratamiento de la información cuantitativa se usaron procedimientos simples de conteo de frecuencias y cálculo de porcentajes a partir de los cuales fueron elaboradas tablas destacando género de autores, instituciones que representan y países de origen. Para la información cualitativa se realizó un análisis de contenido de cada uno de los resúmenes identificando la temática principal que los autores expusieron en sus respectivos trabajos. El resultado de dichos análisis se desarrolla a continuación.

\section{Histórico de la Producción Científica difundida en Paradigma.}

El cuadragésimo aniversario de la revista Paradigma, está precedido por una historia muy particular. El 30 de julio de 1980 se produjo su primera edición y, desde ese momento se ha mantenido activa, de manera continuada, hasta el presente (2020), periodo durante el cual se han publicado 677 artículos número éste que, debido a las 42 contribuciones incluidas en el presente número, alcanza el total de 719 que, durante estas cuatro décadas, están distribuidos en 41 ediciones normales o extraordinarias. Toda la producción por décadas de la revista Paradigma se muestra en la Tabla 1. 
Tabla 1. Distribución de artículos por volúmenes publicados

\begin{tabular}{|c|c|c|c|c|}
\hline $\begin{array}{c}\text { DÉCADA - } \\
\text { VOLÚMENES }\end{array}$ & AÑO & $\mathrm{N}^{\mathrm{o}} 1$ & $\mathrm{~N}^{\mathrm{o}} 2$ & $\begin{array}{c}\text { TOTAL ARTÍCULOS POR } \\
\text { VOLUMEN }\end{array}$ \\
\hline \multirow{10}{*}{$\begin{array}{l}1^{\text {era }} \\
\text { I - X }\end{array}$} & 1980 & 6 & 4 & 10 \\
\hline & 1981 & 6 & - & 6 \\
\hline & 1982 & 6 & - & 6 \\
\hline & 1983 & 6 & - & 6 \\
\hline & 1984 & \multicolumn{2}{|c|}{10} & 10 \\
\hline & 1985 & \multicolumn{2}{|c|}{9} & 9 \\
\hline & 1986 & \multicolumn{2}{|c|}{11} & 11 \\
\hline & 1987 & \multicolumn{2}{|c|}{15} & 15 \\
\hline & 1988 & 5 & 5 & 10 \\
\hline & 1989 & 7 & 6 & $13(96)$ \\
\hline \multirow{7}{*}{$\begin{array}{c}2^{\mathrm{da}} \\
\mathrm{XI}-\mathrm{XX}\end{array}$} & 1990 & $1(*)$ & 3 & 4 \\
\hline & 1991 & \multicolumn{2}{|c|}{8} & 8 \\
\hline & 1992 & 6 & 4 & 10 \\
\hline & 1993-1996 & \multicolumn{2}{|c|}{11} & 11 \\
\hline & 1997 & 6 & 6 & 12 \\
\hline & 1998 & 7 & 13 & 20 \\
\hline & 1999 & 10 & 8 & $18(83)$ \\
\hline \multirow{10}{*}{$\begin{array}{c}3^{\text {era }} \\
\text { XXI - XXX }\end{array}$} & 2000 & 7 & 7 & 14 \\
\hline & 2001 & 6 & 5 & 11 \\
\hline & 2002 & 6 & 8 & 14 \\
\hline & 2003 & 8 & 9 & 17 \\
\hline & 2004 & 10 & 9 & 19 \\
\hline & 2005 & 9 & 9 & 18 \\
\hline & 2006 & 13 & 12 & 25 \\
\hline & 2007 & 11 & 11 & 22 \\
\hline & 2008 & 11 & 12 & 23 \\
\hline & 2009 & 11 & 11 & $22(185)$ \\
\hline \multirow{12}{*}{$4^{\text {ta }}$ XXXI - XL } & 2010 & 10 & 10 & 20 \\
\hline & 2011 & 8 & 10 & 18 \\
\hline & 2012 & 8 & 8 & 16 \\
\hline & 2013 & 8 & 9 & 17 \\
\hline & 2014 & 8 & 11 & 19 \\
\hline & 2015 & 11 & 10 & 21 \\
\hline & 2016 & 11 & 15 & 26 \\
\hline & 2017 & 18 & 21 & 39 \\
\hline & 2018 & 25 & 15 & 40 \\
\hline & 2018 EXTRA & 16 & 19 & 35 \\
\hline & 2019 & 22 & 13 & 35 \\
\hline & 2019 EXTRA & 12 & - & $12(298)$ \\
\hline XLI & 2020 EXTRA & 15 & & 15 (TOTAL: 677) \\
\hline
\end{tabular}

Fuente: Datos de la investigación 
Como se evidencia en la Tabla 1, a lo largo de su trayectoria histórica, la producción de Paradigma se ha ido incrementando sostenidamente, mostrando un mayor crecimiento del número de publicaciones en las dos últimas décadas. Se estima que esto está asociado con el aumento de los estudios de postgrado, así como también con el reconocimiento social de la producción de ciencia, lo cual a su vez ha propiciado que la comunicación científica, de acuerdo con Barrere (2019), se haya transformado en una acción ineludible para todos los agentes vinculados con los procesos de producción de conocimiento.

\section{Hallazgos a partir de los indicadores de la Edición Cuadragésimo Aniversario:} 1980-2020 (Vol. XLI, junio de 2020)

A continuación, se muestran algunos de los indicadores relativos a los artículos incluidos en la edición conmemorativa del cuadragésimo aniversario de la Revista Paradigma. Los aspectos considerados son: género de los autores, país de procedencia e instituciones a las cuales están vinculados, las temáticas en las que fueron distribuidos los artículos y los aportes de cada uno de éstos.

\section{Género de autores}

En lo que parece ser una tendencia en las revistas humanísticas en general y educativas en particular, también en la edición del cuadragésimo aniversario Paradigma, predominan los artículos escritos por mujeres (Tabla 2)

\begin{tabular}{|c|c|c|}
\hline PAÍS & MUJERES & HOMBRES \\
\hline Argentina & $3 \mathrm{M}$ & $1 \mathrm{H}$ \\
\hline Brasil & $18 \mathrm{M}$ & $14 \mathrm{H}$ \\
\hline Chile & $6 \mathrm{M}$ & $5 \mathrm{H}$ \\
\hline Colombia & $1 \mathrm{M}$ & $1 \mathrm{H}$ \\
\hline Cuba & $2 \mathrm{M}$ & - \\
\hline EEUU & $1 \mathrm{M}$ & - \\
\hline España & $7 \mathrm{M}$ & $3 \mathrm{H}$ \\
\hline Italia & - & $1 \mathrm{H}$ \\
\hline Japón & - & $1 \mathrm{H}$ \\
\hline México & $2 \mathrm{M}$ & - \\
\hline $\begin{array}{c}\text { Puerto } \\
\text { Rico }\end{array}$ & $1 \mathrm{M}$ & - \\
\hline Venezuela & $12 \mathrm{M}$ & $6 \mathrm{H}$ \\
\hline TOTAL: & $53 \mathrm{M}$ & $32 \mathrm{H}$ \\
\hline
\end{tabular}

Fuente: Datos de la Investigación 
En relación al género podemos apreciar, según los datos mostrados en la Tabla 2, que es más alto el número de autoras con un 62,35 \%, que de autores, con un 37,65 \%, lo cual revela la calidad de la representación de la mujer en una determinada área disciplinar (Giner-Soriano, 2019), siendo ello evidente en el caso de los profesionales de educación universitaria donde los estereotipos de género están ligadas a percepciones permanentes de los papeles asignados a hombres y mujeres; en los cuales, a decir de los datos persiste la creencia de que las mujeres parecen ser más adecuadas para ejercer la labores de educar (García Villanueva; Ávila Rodríguez; Vargas Pérez; Hernández Ramírez, 2015); destacándose en el rol que tradicionalmente se la atribuyó a la mujer como madre educadora, incidiendo así en la feminización de la carrera docente-investigador (Verardo, 2017).

\section{Instituciones y países presentes en la edición.}

La diversidad en la representación de instituciones universitarias y sus autores es uno de los criterios que se tiene para valorar las revistas científicas (Packer; Prat; Luccisano; Montanari; Santos; Menghini, 2006). Estos parámetros en relación con la edición aniversario de Paradigma son mostrados en el Cuadro 1.

Cuadro 1. Representación Institucional y País de Origen de los autores.

\begin{tabular}{|l|l|}
\hline \multicolumn{1}{|c|}{ REPRESENTA CIÓN INSTITUCIONAL } & $\begin{array}{c}\text { PAÍ́ DE } \\
\text { ORIGEN DE LOS } \\
\text { AUTORES }\end{array}$ \\
\hline 1. Instituto Superior de Formación Docente Simón Bolívar, (ISFDSB) & Argentina (4) \\
2órdoba. & \\
2. Universidad Nacional de General Sarmiento (UNGS) & \\
3. Universidad Nacional del Nordeste (UNNE) & \\
4. Universidad Nacional de Rosario (UNR) & \\
\hline 1. Colegio Estadual Donato Coutinho de Abreu (Goiás) & \\
2. Instituto Federal de Mato Grosso (IFMT) & \\
3. Pontifícia Universidade Católica de São Paulo (PUC-SP) & \\
4. Universidade Anhanguera de São Paulo (UASP) & \\
5. Universidade Cidade de São Paulo (UNICID) & \\
6. Universidade Estadual de Maringá (UEM) & \\
7. Universidade Estadual do Paraná (UNESPAR) & \\
8. Universidade Estadual Paulista "Júlio de Mesquita Filho" \\
9. Universidade Federal de Ouro Preto (UFOP) & \\
10. Universidade Federal de Santa Catarina (UFSC) & \\
11. Universidade Federal do Pará (UFPA) & \\
12. Universidade Federal do Paraná (UFP) & \\
13. Universidade Federal do Rio Grande do Norte (UFRN) & \\
14. Universidade Luterana do Brasil (ULBRA) & \\
15. Universidade Tecnológica Federal do Paraná (UTFPR) &
\end{tabular}

Continúa... 
Cuadro 1. Representación Institucional y País de Origen de los autores. (Continuación)

\begin{tabular}{|c|c|}
\hline REPRESENTACIÓN INSTITUCIONAL & $\begin{array}{c}\text { PAÍS DE } \\
\text { ORIGEN DE LOS } \\
\text { AUTORES }\end{array}$ \\
\hline $\begin{array}{l}\text { 1. Corporación Municipal de Quilpué, } \\
\text { 2. Pontificia Universidad Católica de Valparaíso (PUCV) } \\
\text { 3. Universidad Católica del Maule } \\
\text { 4. Universidad Católica de Temuco } \\
\text { 5. Universidad de Bío-Bío } \\
\text { 6. Universidad de Los Lagos (UdL); } \\
\text { 7. Universidad de Talca }\end{array}$ & Chile (7) \\
\hline $\begin{array}{l}\text { 1. Universidad Antonio Nariño } \\
\text { 2. Universidad Distrital Francisco José de Caldas }\end{array}$ & Colombia (2) \\
\hline Universidad de Camagüey Ignacio Agramonte Loynaz & Cuba (1) \\
\hline Harleyville-Ridgeville Middle School; South Carolina & Estados Unidos (1) \\
\hline $\begin{array}{l}\text { 1. Universidad de Granada } \\
\text { 2. Universidad de Barcelona }\end{array}$ & España $(2)$ \\
\hline Universidad de Bologna (UB) & Italia (1) \\
\hline University of Tsukuba (UT) & Japón (1) \\
\hline $\begin{array}{l}\text { 1. Universidad Autónoma de Guerrero (UAG) } \\
\text { 2. Universidad de Guadalajara (UDG) }\end{array}$ & México (2) \\
\hline Universidad de Puerto Rico (UPR) & Puerto Rico (1) \\
\hline $\begin{array}{l}\text { 1. Universidad Bicentenaria de Aragua (UBA) } \\
\text { 2. Universidad Central de Venezuela (UCV) } \\
\text { 3. Universidad de Carabobo (UC) } \\
\text { 4. Universidad del Zulia (LUZ) } \\
\text { 5. Universidad José Antonio Páez (UJAP) } \\
\text { 6. Universidad Nacional Abierta (UNA) } \\
\text { 7. Universidad Pedagógica Experimental Libertador (UPEL) } \\
\text { 8. Universidad Simón Bolívar (UBA) }\end{array}$ & Venezuela (8) \\
\hline
\end{tabular}

Fuente: Datos de la Investigación.

Como muestra la información vertida en el Cuadro 1, la afiliación institucional, así como el origen geográfico de los autores, evidencia la amplitud de esta edición. En efecto, los autores proceden de 12 países; dos europeos (España e Italia), Estados Unidos, Japón; y ocho países de la región latinoamericana. Los 85 autores $(53 \mathrm{M}$ y $32 \mathrm{H})$ están afiliados a 45 instituciones, siendo 43 de ellas instituciones de educación superior.

\section{Temáticas abordadas en la edición por el cuadragésimo aniversario de Paradigma}

El estudio de los temas es importante para saber los énfasis en las producciones y su vigencia (Villegas, González, Bolívar, Hernández, 2004). Las temáticas desarrolladas en los 42 trabajos que componen esta edición aniversario, están todas ligadas a la educación y la formación de profesores en las más diversas áreas y disciplinas, desde perspectivas fundantes 
de las ciencias humanas y asuntos socio políticos globales, hasta la realización de investigaciones prácticas en varios ámbitos, tales como la enseñanza de las ciencias, las matemáticas, las tecnologías digitales, la educación ambiental, el análisis del discurso, entre otras; a partir de esa caracterización, los artículos fueron organizados en secciones según las temáticas tratadas en ellos (Cuadro 2).

Cuadro 2. Secciones y Temáticas abordadas en la Edición del Cuadragésimo aniversario

\begin{tabular}{|l|l|}
\hline SECCIÓN & \multicolumn{1}{|c|}{ TEMÁTICA } \\
\hline $1^{\text {ra }}$ Sección & Prospectivas de la producción científica en Ciencias Humanas \\
\hline $2^{\text {da }}$ Sección & Formación de los profesores que enseñan Matemática. \\
\hline $3^{\text {ra }}$ Sección & Enseñanza de las ciencias y el desarrollo de competencias científicas \\
\hline $4^{\text {ta }}$ Sección & Reflexiones sobre el uso didáctico de las TIC' \\
\hline $5^{\text {ta }}$ Sección & Experiencias de enseñanza y aprendizaje de entidades matemáticas específicas \\
\hline $6^{\text {ta }}$ Sección & Educación Estadística \\
\hline $7^{\text {ma }}$ Sección & Asuntos socio políticos globales \\
\hline $8^{\text {va }}$ Sección & Historia de la Educación Matemática \\
\hline $9^{\text {na }}$ Sección & La Investigación en la UPEL \\
\hline
\end{tabular}

Fuente: Datos de la investigación

\section{Áreas temáticas y características de los artículos}

Como se planteó en el subtítulo anterior, los artículos se organizaron en áreas temáticas en función de los títulos y el contenido del resumen; proceso del cual surgió nueve secciones, cuyas características y naturaleza del trabajo se desarrollan a seguir.

La primera sección está compuesta por diez artículos que desarrollan temáticas amplias y prospectivas asociadas con diferentes aspectos de la producción científica en el ámbito de las ciencias humanas. Así con los avances observados en los recientes desarrollos teóricos del Enfoque Histórico Cultural; es a esto a lo que se refiere la primera autora Wanda Rodríguez, de la Universidad de Puerto Rico (Recinto Rio Piedras) quien examina el estado actual de la relación entre el origen y desarrollo de las funciones metales superiores y la educación, en función de un movimiento revisionista que actualmente hace énfasis en aspectos ignorados o minimizados en las interpretaciones dominantes de la obra de Vygotski. La autora concluye afirmando que los desarrollos contemporáneos en el enfoque histórico-cultural pueden contribuir a una educación que responda más efectivamente a las demandas que las transformaciones históricas, sociales y culturales actuales han impuesto sobre ella. 
La autora del segundo de los trabajos incluidos en esta primera sección es Maria Aparecida Viggiani Bicudo de la Universidade Estadual Paulista - UNESP, Câmpus de Rio Claro; São Paulo, Brasil quien aporta un ensayo en el cual trata "cuestiones de fondo relativas a la investigación fenomenológica en educación" entre las cuales destacan las siguientes nociones: "lo dado, la subjetividad, la intersubjetividad, y la objetividad, que son temas que se entrelazan cuando se busca comprender la educación y la investigación desde el abordaje fenomenológico".

A continuación, se encuentra el trabajo de Maria da Conceição Passeggi, vinculada con la Universidade Federal do Rio Grande do Norte y la Universidade Cidade de São Paulo, Brasil. Esta autora discurre sobre las aperturas epistemológicas que son propiciadas por la investigación cualitativa en Educación, enfatizando los abordajes narrativos, entre los cuales resalta: las historias de vida en formación, la pesquisa biográfica en Educación y la pesquisa (auto)biográfica y las trayectorias que tales abordajes describieron en Brasil. La autora examina sus diferencias y semejanzas, así como también los principios epistemológicos subyacentes a tres tipos de apuestas que se infieren de esos tres abordajes, sobre esa base propone el que denomina paradigma narrativo-autobiográfico.

A esa primera sesión, también pertenece el aporte, Juan D. Godino y María Burgos, de la Universidad de Granada (España) quienes se cuestionan ¿Cómo enseñar las matemáticas y ciencias experimentales? Indicando que "a pesar de los enormes esfuerzos de investigación que se vienen realizando, este problema sigue abierto". Los autores abordan la cuestión examinando el dilema entre "enseñanza transmisiva" y "aprendizaje indagativo" utilizando los supuestos y herramientas teóricas del Enfoque Ontosemiótico. Su conclusión es que "la optimización del aprendizaje y el logro de una acción didáctica idónea requiere entretejer de manera dialéctica y compleja los momentos de transmisión del conocimiento por el profesor con los momentos de indagación del estudiante."

Seguidamente, en la misma sección señalada antes, está el trabajo de Vicenç Font, Adriana Breda, de la Universitat de Barcelona (España) y Luis R. Pino-Fan, de la Universidad de Los Lagos (Osorno, Chile) en el cual explican la evolución de una agenda de investigación que tiene como foco el estudio de la complejidad de los objetos matemáticos y comentan su experiencia de formación de profesores en la cual se ha de tener en cuenta "la complejidad del objeto matemático a enseñar". Seguido, se presenta el texto de Bruno D'Amore de la 
Universidad de Bologna (Italia) y de la Universidad Distrital Francisco José de Caldas (Bogotá, Colombia) a la cual está adscrita también Martha Isabel Fandiño Pinilla, "presentan y sintetizan diversas reflexiones sobre las bases teóricas del Enfoque ontosemiótico del conocimiento matemático y de la enseñanza de la matemática, en el marco general de la progresiva consolidación de la Didáctica de la Matemática como disciplina científica."

El siguiente trabajo que compone esta sección es un ensayo de Ubiratan D’Ambrosio quien realiza "reflexiones sobre los movimientos STEM y STEAM, desde la perspectiva de la Etnomatemática". Primeramente, ubica el tema "en el contexto de la urgencia que está experimentando la humanidad, debido a la aparición de un nuevo virus corona que generó el COVID-19, que ha propiciado la pandemia que el mundo ha sufrido desde fines de 2019 y que para el momento de la aparición de este número de la Revista Paradigma (junio de 2020) ha causado cientos de miles de muertes en casi todos los países. Posteriormente, ofrece una visión crítica del movimiento STEM que generó la propuesta STEAM, que difiere de STEM al incluir la A de Artes. Con respecto a este cambio, el autor del ensayo, desde la Filosofía de las Matemáticas, se pregunta si hay un lugar para la ficción y la fantasía en las Matemáticas, que son esenciales en las Artes. Para responder esto se necesitan algunas preguntas preliminares que el autor responde desde el Programa de Etnomatemáticas y plantea su perspectiva personal para introducir el concepto de STEAM."

Abordando un tema muy sensible y al mismo tiempo importante como lo es la Educación Inclusiva, Sani de Carvalho Rutz da Silva, Renata da Silva Dessbesel, de la Universidade Tecnológica Federal do Paraná (Ponta Grossa, Brasil) y Elsa Midori Shimazaki, de la Universidade Estadual de Maringá (Brasil), nos ofrecen “un panorama de las investigaciones referentes la enseñanza de las matemáticas en la educación de personas sordas" en cuyo desarrollo utilizaron la "Methodi Ordinatio, para la selección y análisis de los artículos disponibles en la base de datos Scopus, Scielo, DOAJ y ERIC'. Estas investigadoras concluyen afirmando que "la educación de los sordos se presenta en un ambiente de muchas posibilidades $y$, por lo tanto, diversos factores interfieren en el proceso de enseñanza y aprendizaje de las matemáticas de estas personas en el aula."

A continuación, Raimundo Olfos y Soledad Estrella, de la Pontificia Universidad Católica de Valparaiso (Chile) y Masami Isoda, de la University of Tsukuba (Japón), presenta un conjunto de hallazgos y avances logrados durante más de una década de investigaciones 
sobre el Estudio de Clases (Lesson Study), como metodología de desarrollo profesional docente, llevadas a cabo en Chile. Como resultado de su experiencia, estos autores afirman que es necesaria una "mayor comprensión a nivel político de una concepción ampliada del Estudio de Clases, como vehículo efectivo en la transformación del aula de clases, la escuela y el sistema escolar."

Esta primera sección se cierra con el trabajo de Adriana Bolívar, de la Universidad Central de Venezuela, quien presenta su estudio sobre el discurso académico, usando como referencia teórica el Análisis Interaccional del Discurso, a través del entrenamiento ofrecido a profesores universitarios sobre los pormenores asociados con la escritura de un buen artículo científico en Educación, acción ésta que constituye un complejo "proceso que abarca en la teoría varios planos: a) la definición de artículo científico, b) los tipos de textos que aceptan las revistas como productos de la investigación científica, c) las tradiciones discursivas en educación, y d) las estrategias de los investigadores para cumplir con el requisito de escribir un buen artículo cientifico".

La segunda sección, compuesta por cuatro trabajos, está dedicada a la formación de los profesores que enseñan Matemática. El primero de ellos, Hugo Parra-Sandoval, de la Universidad del Zulia (Venezuela), considera un aspecto clave en este asunto: la formación que deben tener quienes se encargan de formar a los futuros profesores de esta disciplina, es decir, la formación de los formadores, haciendo énfasis en que para ello se requiere de un conocimiento especializado. Parra concluye su trabajo proponiendo una agenda de investigación sobre dos aspectos que considera esenciales: la problematización y el conocimiento del formador de profesores.

En el segundo de los artículos que componen esta sección, Nancy Montes de Oca Recio, de la Universidad de Camagüey Ignacio Agramonte Loynaz (Cuba), muestra algunos resultados teóricos relacionados con la formación didáctico-matemática de los docentes de Matemática, generados "a partir de las insuficiencias detectadas en el desempeño de estos docentes en el ejercicio de la profesión, en relación con nociones básicas de Geometría Plana y de Álgebra Lineal, gestión de demostraciones matemática, promoción de la valoración positiva de la Matemática, y comunicación matemática. Las bases teóricas del estudio corresponden al enfoque histórico cultural del desarrollo humano y los enfoques ontosemiótico, comunicativo y contextualizado de la enseñanza-aprendizaje de la Matemática”. 
Natalia Sgreccia, de la Universidad Nacional de Rosario (Argentina), autora del tercero de los artículos que componen la segunda sección, realiza una pesquisa en el repositorio histórico (1980-2019) de la Revista Paradigma identificando los artículos que abordan la formación de profesores de Matemática, analizándolos en función de su propia línea de pesquisa. Sgreccia desarrolla su trabajo compartiendo "una síntesis del contenido de cada una de las obras ensayando, además, relaciones conceptuales, a modo esquemático, entre las mismas. Finalmente, se comenta brevemente la línea de trabajo actual de la autora y su equipo de investigación en Argentina, que se encuentra en correspondencia con el tópico abordado".

Para cerrar esta sección, Silvia N. Retamal Cisterna, Luis R. Pino-Fan, de la Universidad de Los Lagos, (Osorno, Chile) y Sonia Salas-Salinas, de la Corporación Municipal de Quilpué (Chile), comparten sus reflexiones sobre el aprendizaje de la Matemática fuera del espacio escolar y su incidencia sobre la formación de profesores, generadas a partir de la asunción de una visión sociopolítica de la enseñanza de la matemática escolar en el escenario chileno que les permitió "entender los mecanismos por los que se reproducen desigualdades desfavorables para los estudiantes más vulnerables de nuestra sociedad y con ello identificar directrices para contribuir con la justicia social desde el ámbito educativo".

La tercera sección está conformada por cinco artículos y se dedica a la enseñanza de las ciencias y el desarrollo de competencias científicas de estudiantes de diferentes niveles educativos.

En el primero se detalla el proceso de construcción de un instrumento para evaluar mediante juegos, competencias matemáticas y científicas del alumnado que inicia educación primaria, desarrollado por María José Espigares Gámez, Alicia Fernández Oliveras y María Luisa Oliveras Contreras de la Universidad de Granada (España). El estudio tuvo como premisa que "existen capacidades matemáticas y científicas en los jugadores, que se pueden poner de manifiesto cuando se juegan, con rigor y éxito, juegos con potencialidades matemáticas y científicas. Para determinar éstas, se realizó el análisis de un conjunto de juegos tradicionales pertenecientes a diversas culturas y países del mundo, desde una perspectiva Etnomatemática", y en el mismo fueron analizadas "las potencialidades matemáticas y científicas de los juegos, mediante un análisis de contenido de las reglas de juego y un análisis etnográfico de los materiales y el contexto del juego, estableciendo un catálogo de 40 juegos válidos para aprender y evaluar matemáticas y ciencias al ser jugados". 
En el segundo, Everton Bedin, de la Universidade Federal do Paraná (Brasil) y José Claudio Del Pino, de la Universidade do Vale do Taquari (Brasil), presentan su constructo teórico vinculado con la movilización de competencias cognitivas en la enseñanza de las ciencias; su planteamiento central es que “cuando el Aprender por la Investigación Centrada en el Alumno (APCA), se desarrolla a partir del interés del estudiante y de la inserción activa de éste en el aula, le permite la movilización de competencias para suformación crítica yética”.

El tercero de los trabajos incluidos en esta sección, Letícia Ferreira y Lucila Akiko Nagashima de la Universidade Estadual do Paraná, Paranavaí (Brasil), muestran el aporte que la literatura infantil, particularmente las fábulas, proporciona a la enseñanza de la ciencia en los primeros años de la Educación Fundamental. Su pregunta clave fue "¿Cómo pueden los maestros asociar la enseñanza de las ciencias con las narraciones literarias trabajadas en el aula?" Para tratar de responderla articularon "a la literatura infantil (particularmente las fábulas) algunas de las características de la enseñanza de animales y ciencias basadas en referencias teóricas de literatura y educación antropológica".

En el cuarto artículo se aborda el papel que desempeña la investigación científica en la formación de estudiantes universitarios. Para ello, las autoras Ligia Sánchez, Maidelis Herrera, de la Universidad de Carabobo, Campus La Morita (Venezuela) y Mairene Sánchez, de la Universidad Bicentenaria de Aragua (Venezuela) realizaron una indagación sobre "la resistencia que tienen los estudiantes universitarios de involucrase en la actividad investigativa durante su proceso de formación inicial". Uno de sus hallazgos fue que "Los estudiantes tienen poco conocimiento respecto a la actividad científica que se desarrolla en la universidad, lo cual limita su participación e involucramiento en dichas actividades y genera cierta aprehensión y temor producto de miedos e inseguridades, ya que la investigación se ve como algo reservado para los elegidos". Sin embargo, al mismo tiempo consideran que "ejecutar el proyecto de investigación, del Trabajo Especial de Grado, es relevante en su formación profesional, por su utilidad académica y su potencial para fortificar el conocimiento disciplinar".

Esta sección se cierra con un trabajo en el cual son examinadas las concepciones previas de alumnos de educación secundara en relación con Educación Ambiental, EA, desarrollando la construcción de un horno solar como práctica educativa. Los autores del trabajo Weslaine da Silva Santos y Marcelo Franco Leão, del Instituto Federal de Mato Grosso (IFMT; Confresa, Brasil), analizaron "las percepciones de los estudiantes de una clase de secundaria en una 
escuela pública en Mato Grosso sobre EA”, así como también desarrollaron "una actividad experimental como una acción práctica sostenible. Los resultados del estudio "revelaron las percepciones sobre el concepto de EA y el medio ambiente, los problemas ambientales actuales, la participación en acciones ambientales, la evaluación de la intervención realizada, las contribuciones al pensamiento crítico y el aprendizaje construido”

La cuarta sección está formada por sólo dos artículos; en el primero de ellos, Fábio Douglas Farias de la Universidade Cidade de São Paulo (Brasil), Douglas da Silva Tinti de la Universidade Federal de Ouro Preto (Brasil) y Ana Lúcia Manrique, de la Pontifícia Universidade Católica de São Paulo (Brasil), disertan sobre el Modelo f @ r, construido a partir de una reflexión conjunta realizada por docentes que enseñan matemáticas después de haber participado en un proceso de capacitación con tecnologías;

El segundo contiene una reflexión sobre el uso didáctico de las TIC’S en las Universidades. Los autores, Haydee Guillermina Páez, de la Universidad José Antonio Páez (Venezuela), Nolberto Goncalves Rodríguez, de la Universidad de Carabobo (Venezuela) y Evelyn Cristina Arreaza Páez, de la Harleyville-Ridgeville Middle School ( South Carolina, EE UU), se preguntan si "transcurridas dos décadas de haber sido establecido como prioridad el uso de la internet en las instituciones de educación superior venezolanas, el uso didáctico de las TIC responde a una expectativa o aspiración y si ésta, es personal o institucional”. El estudio les permitió constatar que existe una "generalizada predisposición negativa de docentes y estudiantes hacia el uso de las TIC como recurso para mediar procesos didácticos en la sociedad actual, lo cual resalta el importante papel que la afectividad digital podría desempeñar para superar esta situación”.

La quinta sección, compuesta por cinco contribuciones, incluye experiencias de enseñanza y aprendizaje de entidades matemáticas específicas en Educación Superior.

En el primero, Carmen Teresa Kaiber y Priscila Augusta de Quadros Scott Hood de la Universidade Luterana do Brasil (Canoas, Rio Grande do Sul), examinan los errores en los que incurren los estudiantes en el aprendizaje del Cálculo Diferencial e Integral y afirman el potencial que su análisis tiene para identificar las dificultades que manifiestan los estudiantes al estudiar Cálculo Diferencial e Integral a nivel universitario, especialmente las que “están vinculadas a aspectos conceptuales relacionados con la resolución de derivadas por definición 
por límite, así como a procedimientos relacionados con conceptos y propiedades elementales dentro del ámbito de Matemáticas”.

En el segundo trabajo, Mónica Campos, del Instituto Superior de Formación Docente Simón Bolívar (Córdoba, Argentina) y Mabel Rodríguez, de la Universidad Nacional de General Sarmiento (Los Polvorines, Argentina), comparten su estudio sobre la aprehensión conceptual de las inecuaciones, realizado en el marco del Enfoque Cognitivo en Educación Matemática, asumiendo explícitamente la teoría de las representaciones semióticas de Raymond Duval, con la participación de "estudiantes del primer año de la formación de profesores de matemática en una institución argentina" El estudio prestó atención principalmente a los "registros de representación semiótica que estudiantes de primer año ponen en juego al resolver inecuaciones y al expresar sus conjuntos solución" así como también al "grado de aprehensión conceptual alcanzado luego de la enseñanza recibida".

El tercer trabajo de esta sección es un aporte de Olga Lidia Pérez González, de la Universidad de Camagüey "Ignacio Agramonte Loynaz” (Cuba) quien se refiere a la formación y desarrollo conceptual en el Cálculo Diferencial y el Álgebra Lineal en las carreras de Ingeniería. Sus formulaciones están fundamentadas en una investigación a partir de la cual fueron diseñadas algunas estrategias relativas a la didáctica de esas dos disciplinas.

En el cuarto artículo, Marcela Parraguez, de la Pontificia Universidad Católica de Valparaíso (Chile) y Guadalupe Vera-Soria, de la Universidad de Guadalajara (México), dan cuenta de un estudio de casos llevado a cabo en un contexto universitario sobre los modos de Pensamiento Sintético y Analítico que los estudiantes ponen en juego para la comprensión del concepto de Base en el Espacio Vectorial $\mathrm{R}^{2}$; las autoras describieron el proceso de "construcción del significado del acto de comprender dicho concepto, cuando se articulan tres modos de pensarlo: sintético-geométrico, analítico-aritmético y lo analítico-estructural" y encontraron "elementos clave para alcanzar la comprensión de Base en $R^{2}$ como un sistema conceptual'.

El trabajo de cierre de esta sección fue producido por Claudia Lisete Oliveira Groenwald, de la Universidade Luterana do Brasil (Canoas, Rio Grande do Sul) a partir de un Diseño Instruccional desarrollado con cinco estudiantes de pregrado en Matemáticas con el tema expresiones numéricas. Se trató de un estudio de caso en el que "se aplicó una situación 
didáctica con el tema Expresiones numéricas, adecuada para estudiantes en el sexto año de la escuela primaria".

La sexta sección está dedicada a la Educación Estadística. La conforman tres trabajos. En el primero de ellos, Nelly León Gómez, de la Universidad Pedagógica Experimental Libertador (Instituto Pedagógico de Maturín, Venezuela), examina los alcances de la Enseñanza de la Estadística a través de la Investigación en la Educación Media en ese país suramericano. El asunto central abordado por la autora se refiere a "pertinencia de una enseñanza de la Estadística a través de la investigación con miras a la formación de un ciudadano crítico”. En primer lugar examina el estatuto de la Estadística en cuanto ciencia: luego cuestiona "la forma como se enseña Estadística en Venezuela en Educación Media, apegada a la tradición calculista y procedimental", procurando evidenciar "las limitaciones y las oportunidades de los docentes para una enseñanza/aprendizaje de la disciplina con la aplicación de la investigación estadística a través de proyectos de aprendizaje”. Esto implicaría cambios en los procesos de formación docente que "conlleven a adoptar un modelo que contemple por una parte el conocimiento conceptual y pedagógico para la enseñanza y por otra el desarrollo del pensamiento estadístico de los propios docentes, sin olvidar el componente afectivo de la Educación"

En el segundo, Carmen Batanero, Antonio Francisco Roldán López de Hierro y Rocío Álvarez Arroyo, de la Universidad de Granada (España), evaluaron las dificultades que confrontan los estudiantes en la construcción de intervalos de confianza, uno de los conceptos clave en la Educación Estadística; para ello, analizaron “los pasos requeridos para resolver un problema abierto tomado de las pruebas españolas de acceso la universidad y compararon las soluciones aportadas por 58 estudiantes de Bachillerato y 57 de Psicología, después de haber estudiado el tema".

Por último, se presenta el estudio realizado por Danilo Díaz-Levicoy, de la Universidad Católica del Maule (Talca, Chile), Lizzet Morales-Garcia, de la Universidad Autónoma de Guerrero (México) y Francisco Rodríguez-Alveal, de la Universidad de Bío-Bío (Chillán, Chile), sobre las actividades en que intervienen las medidas de tendencia central en libros de texto de Educación Primaria utilizados en México. Para ello desarrollaron un análisis de contenido en libros de primaria usados en ese país. Los investigadores encontraron " $e l$ predominio de la media aritmética y la moda, la tarea de calcular, el uso de las 
representaciones de listado de datos y tabla de datos y el contexto personal [...] se observa la necesidad de aumentar la cantidad de actividades sugeridas en los libros de texto".

En la séptima sección se incluyen cuatro artículos que abordan asuntos globales. El primero está relacionado con las funciones de la escuela secundaria brasileña en el contexto del ajuste neoliberal y de la crisis del capital. Este trabajo fue desarrollado por Neide de Almeida Lança Galvão Favaro, Priscila Semzezem y Cleissiane Aguido Gotardo, de la Universidade Estadual do Paraná (Campus Paranavaí, Brasil) quienes analizan "las funciones que asume la escuela secundaria brasileira con vistas a la Ley $N^{\circ} 13.415 / 2017$, que cambia la escuela secundaria brasileña, insertándola en el marco de las políticas neoliberales y en medio de la fase actual de la reestructuración del capital mundial'. Por su parte, Karina Heredia y Julio González Bello, de la Universidad de Carabobo (Venezuela), desarrollan la noción de Autonomía Deontológica, como uno de los principios básicos que debe fundamentar la práctica professional de los Orientadores, en apego a lo establecido en la Declaración Universal sobre Bioética y Derechos Humanos.

El tercero de los trabajos es de autoría de Marling Rosario Brito, Freddy Rojas Velásquez, de la Universidad Simón Bolívar (Caracas, Venezuela), junto con Juan Bolívar López, de la Universidad de Talca (Chile) quienes estudiaron la influencia conjunta del Estilo de Educación Parental y el Estilo de Pensamiento de los Estudiantes sobre el Rendimiento Académico de éstos. A partir de los resultados de su pesquisa diseñaron "un Programas de Educación Familiar para padres y de Entrenamiento Didáctico para profesores con la finalidad de fomentar en el estudiante adolescente un mejor proceso de socialización y respuesta a las tareas para su éxito académico”.

El siguiente de los trabajos incluidos en esta sección pertenece a las profesoras María Susana Harringhton Martínez, de la Universidad Pedagógica Experimental Libertador (Núcleo El Mácaro, Venezuela), Lourdes Ángela Díaz Blanca, de la Universidad Pedagógica Experimental Libertador (Mérida, Venezuela) y Ana Cristina Bolívar Orellana, del Instituto de Formación docente Salomé Ureña (San Juan de la Maguana. República Dominicana). Estas tres especialistas en Lingüística, realizaron un estudio relative a las representaciones sociales acerca de los trabajos académicos finales de los cursos de postgrado (disertaciones, tesis, e otros) genéricamente denominados "tesis" que son reflejadas en los memes que se refieren a dichas producciones; "entre las conclusiones destacan que en las representaciones sociales que 
subyacen en los memes la tesis es una meta que solo puede alcanzarse si hay dolor, angustia y sufrimiento de por medio. Dichos resultados plantean la necesidad de revisar la forma en que las instituciones universitarias han orientado el desarrollo de las tesis en pregrado y postgrado".

La séptima sección la cierra el trabajo de Verónica Díaz Quezada (Universidad de Los Lagos) y Cecilia Sanhueza Cartes (Universidad Católica de Temuco), ambas de Chile y quienes muestran un estudio que les permitió elaborar y validar un instrumento para monitorear la adquisición de las denominadas habilidades blandas o transversales en educadores de párvulos; tales habilidades, afirman las autoras, “ [...] son consideradas en la actualidad por la literatura tanto nacional como internacional, imprescindibles en la formación (de dichos educadores), por su complementación con las llamadas competencias duras o genéricas."

La octava sección es sobre Educación Matemática que incluye trabajos sobre historia de esta disciplina y aplicación de la Matemática en la comprensión del comportamiento de la pandemia de COVID-19.

Está compuesta por siete trabajos. El primero remite a las genealogías y visión formativa de grupos de investigación en la historia de la educación matemática en Brasil. Este trabajo es un aporte de Iran Abreu Mendes y Carlos Aldemir Farias da Silva, de la Universidade Federal do Pará (Belém, Brasil), quienes "presentan los resultados de un estudio, basado en la epistemología de Ludwik Fleck y asociado con estudios sobre genealogía, cuyo objetivo fue discutir las genealogías de los grupos de investigación en Historia de la Educación Matemática de Brasil, desde la perspectiva de su visión formativa, y sus estilos y colectivos de pensamiento, con el fin de caracterizar el origen, la evolución y la difusión de las organizaciones sociales de estos grupos de investigación en relación con sus generaciones descendientes. De ese modo, consiguieron identificar una trayectoria de maduración académica en los procesos de formación de los investigadores orientada hacia el fortalecimiento de la constitución epistemológica del campo de investigación en Historia de la Educación Matemática y su ramificación multiplicativa en las diferentes regiones de Brasil.

El segundo trabajo es de la autoría de Wagner Rodrigues Valente, de la Universidade Federal de São Paulo y líder del Grupo de Pesquisa de História da Educação Matemática (GHEMAT), quien muestra algunos resultados que se han obtenido con el desarrollo de un amplio proyecto de investigación sobre el saber profesional del maestro que enseña 
matemáticas; para caracterizar dicho saber, apeló dos categorías: matemáticas a enseñar y matemáticas para enseñar; con base en las relaciones entre estas dos matemáticas, se realiza una construcción teórica sobre el objeto identificado como el saber profesional del profesor que enseña matemáticas.

En el tercero de los trabajos incluidos en esta sección, se comparte una mirada a las producciones en Historia de la educación matemática presentadas en el VII Seminario Internacional de Investigación en Educación Matemática. Este artículo fue producido por Yohana Taise Hoffmann, David Antonio da Costa y Luiz Ricardo Nakamura, de la Universidade Federal de Santa Catarina (Brasil), quienes analizan las comunicaciones expuestas en la edición del mencionado evento correspondiente al año 2018 en el GT15 (Historia de la educación matemática), procurando dar respuesta a las siguientes interrogantes: ¿Cuáles son los grupos de investigación (GI’s) presentes en el GT15? ¿Qué supuestos teóricos y metodológicos se utilizan? ¿Qué temas se están abordando? Para responderlas se apoyaron en los conceptos de colectivo de investigadores (Ludwick Fleck) y campo científico (Pierre Bourdieu).

En el cuarto trabajo de esta octava sección, son presentadas las investigaciones y orientaciones curriculares presentes en la Enseñanza del álgebra en los años iniciales de la Enseñanza Fundamental brasilera. El estudio que generó este artículo fue realizado por Claudianny Amorim Noronha y Luanna Priscila da Silva Gomes, de la Universidade Federal do Rio Grande do Norte (Brasil), quienes examinaron las orientaciones para la enseñanza y el aprendizaje del álgebra en los años iniciales de la Enseñanza Fundamental contenidas en los documentos curriculares oficiales que orienta las prácticas pedagógica en la Educación Básica. Además, realizaron un inventario de los estudios sobre el tema, divulgados en las memorias de uno de los principales eventos brasileros de Educación Matemática.

El quinto trabajo, contiene reflexiones sobre las aproximaciones y distancias entre Hermenéutica y Hermenéutica de Profundidad como marco teórico para los estudios historiográficos en Educación Matemática; sus autores son Alexandre Ausani Huff, Arno Bayer y Fernando Luís de Rosso, de la Universidad Luterana de Brasil (Rio Grande do Sul, Brasil), cuya intención fue "construir los puentes necesarios entre la hermenéutica, la historicidad y la enseñanza de las matemáticas, con el fin de ofrecer subsidios para aquellos que investigan la Historia de las Matemáticas y la Historia de la Enseñanza de las Matemáticas y quienes, a veces, encuentran difícil basar sus estudios con respecto a la escritura. 
El sexto trabajo incluido en esta sección examina prolijamente las ediciones producidas en Caracas del libro Elementos de Geometría de Legendre. Se trata de un nuevo estudio sobre textos antiguos de Matemática, realizado por Walter Beyer, de la Universidad Nacional Abierta (Venezuela), en el cual llevó a cabo un "estudio comparativo de tres ediciones venezolanas de los Elementos de Geometría de André Marie Legendre, libro ampliamente usado en Venezuela. La indagación se realizó siguiendo el método histórico y la metodología de análisis de textos históricos y aporta una descripción del ejemplar de la obra de cada edición, considerando y comparando entre sí diversos elementos constitutivos del texto.

El último de los trabajos incluidos en esta sección trata de las posibilidades de uso de la matemática para la interpretación y comprensión de un problema contemporáneo: la pandemia de COVID-19 ocasionada por la aparición en China, a finales de 2019, de un nuevo coronavirus. Los autores de esta contribución son Juan Nápoles (Argentina) y Osvaldo Rojas (Colombia) quienes abordan el mencionado problema mediante el uso del Modelos SIR, ideado por Kermack y McKendrick (1927), para simular la difusión de una epidemia; los autores discuten "su solución y enfoques y hacen algunas reflexiones educativas, desde las posiciones de la Matemática Realista”.

En la novena sección, dedicada a la Investigación en la UPEL, son comparadas dos estrategias para la enseñanza de la Geometría a futuros profesores de Matemática, por un lado el doblado de papel y por otro el uso de software de Geometría Dinámica. El estudio del cual se derivó este artículo fue llevado a cabo por Martha Iglesias, de la Universidad Pedagógica Experimental Libertador (Instituto Pedagógico de Maracay, Venezuela) y José Ortiz, de la Universidad de Carabobo (Campus La Morita, Venezuela), en el contexto de un curso optativo de Resolución de Problemas Geométricos Asistido por Computadora (RPG-AC) en que participaron 13 futuros profesores de matemática. Fue usado el software de geometría dinámica CabriGéomètre II para "mostrar la construcción con regla y compás de la herramienta triangular, y dejar ver su equivalencia con la construcción a partir del doblado de papel. El estudio realizado puso en evidencia que las actividades y experiencias formativas llevadas a cabo contribuyen a la formación didáctica y matemática de los futuros profesores”. 


\section{Conclusiones}

\section{Producción Científica que circula en Paradigma}

En sus 40 años, Paradigma ha publicado 677 trabajos, sin contar los de la edición del cuadragésimo aniversario; incluyendo ésta se alcanza un total 719 distribuidos en 41 volúmenes, lo cual indica un promedio aproximado de 18 artículos por volumen, lo cual puede ser visto como moderado. Sin embargo, si se considera la producción a partir del 2000, cuando la revista consiguió estabilizarse, el panorama es el siguiente: en la década 2000-2009, fueron publicados 185 trabajos, manteniéndose el promedio de alrededor de 18 artículos por volumen; sin embargo, entre 2010 y 2019, fueron publicados 298 trabajos, lo cual indica un promedio alrededor de 30 artículos por volumen, siendo ello un indicio de la consideración que la comunidad científica tiene hacia la revista a la que le ha sido otorgada la clasificación Qualis $\underline{\text { A2 }}$, herramienta usada por la $\underline{\text { CAPES }}$ (Brasil) para clasificar la producción científica de los programas brasileros de postgrado en lo que se refiere a los artículos publicados en revistas científicas. Además, se mantiene activa en el Directorio de LATINDEX y en la biblioteca

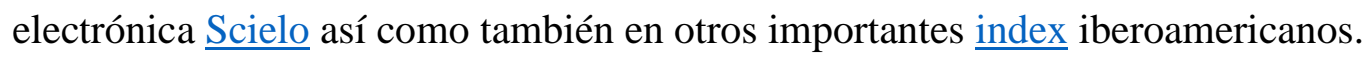

Con base en la información antes expuesta, se puede señalar que la Revista Paradigma constituye una de las publicaciones venezolanas que ha logrado sobreponerse a las múltiples dificultades que sufren las publicaciones científicas latinoamericanas, manteniéndose activa, luego de su primera edición (1980) lo cual supera ampliamente la vida promedio de las revistas latinoamericanas, evidenciando una tendencia hacia su definitiva estabilización, asegurando regularidad y oportunidad en su salida.

\section{Autoría}

Los 42 artículos de la edición por el cuadragésimo aniversario de Paradigma fueron producidos por 85 autores, de los cuales 53 son mujeres y 32 son hombres. Este es otro aspecto interesante ya que la mayoría de las mujeres firmantes de artículos en esta edición son primeras autoras, con lo cual se avanza hacia la superación de la infrarrepresentación femenina en los periódicos científicos del área humanística.

\section{Afiliación Institucional}

En la edición por el cuadragésimo aniversario, están representadas 45 instituciones; 15 de ellas son brasileras y 8 son venezolanas. Estos dos países acumulan aproximadamente el $51 \%$ 
del total. Las 15 instituciones brasileras se distribuyen en prácticamente toda la geografía del país, lo cual acontece de igual manera con las instituciones venezolanas.

\section{Distribución Geográfica}

Las autoras, autores e instituciones representadas en la edición por el cuadragésimo aniversario de la Revista Paradigma, proceden de 12 países localizados en América Latina, América del Norte, Europa y Asia, lo cual es un indicio de la apertura de esta publicación hacia instituciones y países diferentes de Venezuela (sede de la publicación), y una contribución a la visibilidad de la producción científica generada en nuestra región.

En síntesis, teniendo en cuenta lo expuesto, se perciben: la amplia acogida que ha alcanzado la revista, la visibilidad de la misma y la diversidad en la comunicación de las áreas disciplinares relacionada con la formación de profesores.

Finalmente, se resalta que queda pendiente un análisis, utilizando herramientas propias de la cienciometría (Parra, Coutinho \& Pessano, 2019), minucioso y detallado de toda la producción científica contenida en esta publicación a lo largo de sus cuatro décadas de existencia, para lo cual, convidamos a los académicos interesados en realizar este tipo de estudio.

\section{Referencias}

Bárcenas, Ramón (2002). Contexto de descubrimiento y contexto de justificación: un problema filosófico en la investigación científica. Acta Universitaria, 12(2),48-57. ISSN: 0188-6266. Disponible en: https://www.redalyc.org/articulo.oa?id=416/41600206. [Acceso el: 9 de mayo de 2020].

Barrere, R. (Coord.) (2019). El estado de la ciencia. Red de Indicadores de Ciencia y Tecnología -Iberoamericana e Interamericana- RICYT Principales Indicadores de Ciencia y Tecnología Iberoamericanos / Interamericanos. Disponible en: http://www.ricyt.org/wpcontent/uploads/2019/10/edlc2019.pdf . [Acceso el: 9 de mayo de 2020].

Barsky, O. (2014). La evaluación de la calidad académica en debate. Volumen 1. Los rankings internacionales de universidades y el rol de las revistas científicas. Buenos Aires, UAI Editorial-Teseo. Disponible en: https://www.uai.edu.ar/media/109527/la-evaluaci\%C3\%B3nde-la-calidad-acad\%C3\%A9mica-en-debate.pdf. [Acceso el: 9 de mayo de 2020].

Burke, P. (2003). Uma história social do conhecimento: de Gutemberg a Diderot. Rio de Janeiro: Jorge Zahar.

Bufrem, L.; Gabriel Junior, R.; Gonçalves, V. (2010). Dez Anos de Revista Diálogo Educacional (2000-2009): histórico e evolução. Rev. Diálogo Educ., Curitiba, Vol.. 10, n. 29, p. 123-149. Disponible en:

https://www.researchgate.net/publication/321279701_DEZ_ANOS_DE_REVISTA_DIALOGO_EDUCACIONAL_200 0-2009_historico_e_evolucao/citation/download. [Acceso el: 12 de mayo de 2020]. 
Campos, R. (2000). La comunicación científica: ¿arte o técnica? Ars Pharmaceutica, Vol 41 N| 1, p 11-18. Disponible en: http://www.ugr.es/ ars/abstract/41-11-00.pdf. [Acceso el: 9 de mayo de 2020].

Delgado, L.C, Jimenez, C.E, Ruiz,P.R (2009). España y los 25 grandes de la ciencia mundial en cifras (1992-2008). El Profesional de la InformaciónVol, 18, (1) p 81-86. Disponible en: http://eprints.rclis.org/12829/. [Acceso el: 1 de Junio de 2020].

García, A. (2001). Redes Digitales y Exomemoria. Texto presentado como resumen del trabajo de investigación, intitulado, "La memoria subrogada. Mediación, cultura y conciencia en la red digital", durante el I Congreso Ibérico de Comunicación, realizado en Málaga del 7-9 de Mayo de 200. Disponible en: https://abacus.universidadeuropea.es/bitstream/handle/11268/2800/a_gutierrez.pdf?sequence=1. [Acceso el: 9 de Junio de 2020].

García Villanueva, J., Ávila Rodríguez, D., Vargas Pérez, M., \& Hernández Ramírez, C. (2015). Acerca de la feminización de profesiones. Caso: la docencia en preescolar en la Cuidad de México. La ventana. Revista de estudios de género, 5(42), 129-151. Disponible en: http://www.scielo.org.mx/scielo.php?script=sci_arttext\&pid=S1405-94362015000200129\&lng=es\&tlng=es [Acceso el: 08 de junio de 2020]

Giner-Soriano M.; López-Pereiro, A.; Zabaleta-del-Olmo, E.; Pons-Vigués, M.; Morros, R.; Gómez-Lumbreras, A. (2019). Análisis bibliométrico de la autoría femenina en artículos originales en la revista Atención Primaria. 2019. Disponible en: https://reader.elsevier.com/reader/sd/pii/S0212656719304238?token=8EBCD3B3A0B41E545552081CFF7FE [Acceso el: 08 de junio de 2020]

Goncalves, A. Costa-Ramos, L.M.S.V. Figueredo-Castro,R:C (2006) Revistas científicas: Características, funcoes e critérios de qualidade. En Población, D.A, Witter, P.G, Da Silva, J.F.M Comunicacao \& producto científica: Contexto, indicadores e avaliação. Cap 6, p. 165-187. Sao Paulo, SP: Angellara.

González, F. (2008). Revisión del Contenido de la Revista Paradigma (1980-2007). Comunicación oral presentada en el Primer Encuentro Internacional de Revistas Científicas: retos de las revistas científicas para la educación en la sociedad actual, realizado en Bogotá, Colombia, del 7 al 8 de mayo de 2008.

González, F. (2014). Dificultades en la realización de trabajos de investigación: como afrontarlas. Práxis Educacional, [S.1.], v. 11, n. 18, p. 275-300, nov. Disponível em: http://periodicos2.uesb.br/index.php/praxis/article/view/812. Acceso el: 09 jun. 2020.

Haupt, C. (2000). Las revistas científicas latinoamericanas: su difusión y acceso a través de bases de datos. Biblioteca Universitaria (nueva época), 3(2), 122-127. Disponible en: http://dgb.unam.mx/servicios/dgb/publicdgb/bole/fulltext/volIII2/octava.PDF [Acceso el: 08 de junio de 2020]

Kelly, J., Sadeghieh, T., \& Adeli, K. (2014). Peer Review in Scientific Publications: Benefits, Critiques, \& A Survival Guide. EJIFCC, 25(3), 227-243.

Kermack, W. O. and McKendrick, A.G. (1927), Contributions to the Mathematical Theory of Epidemics, Proc. Roy. Soc. A. vol. 115, 700-721.

Lara, C. K., Witter, P. G (1999). Análise de títulos do periódico Paradigma. Em Witter, P.G. (Org.) Produção científica em psicologia e Educação. Cap 6, p.131-149. 
Luchillo, Lucas Jorge. (2014). Revistas científicas: oligopolio y acceso abierto. Revista CTS, $n^{o}$ 40, vol. 14, Febrero de 2019 (pág. 41-79). Disponible en: http://www.revistacts.net/volumen-14numero-40/352-articulos/873-revistas-cientificas-oligopolio-y-acceso-abierto [Acceso: 09 de junio de 2020]

Meadows, A. J. (1999). A comunicação científica. Brasília: Briquet de Lemos.

Meza, M.A. (2005). Estudio métrico revista Cuadernos del Cendes: producción productividad en trece años de publicación. CDC, vol., $22 \mathrm{~N}^{\circ}$ 60, p 173- 180. Disponible en: http://ve.scielo.org/ [Acceso: 09 de junio de 2020]

Mourao, A. O.M.H (1999). Avalicao de producao científica. En Witter P. G. (Org.) Producao científica em Psicología e Educacao . Cap. 1, p 9-22 Sao Paulo, SP, Editore Alínea

Packer, Abel L.; Prat, Ana María; Luccisano, Adriana; Montanari, Fabiana; Santos, Solange; Menghini, Rogério. El modelo SciELO de publicación científica de calidad en acceso abierto. En publicación: Babini, Dominique; Fraga, Jorge CLACSO, Consejo Latinoamericano de Ciencias Sociales. Ciudad de Buenos Aires, Argentina. 2006. pp 191208 ISBN: 987-1183-53-4 Disponible en la World Wide Web: http://bibliotecavirtual.clacso.org.ar/ar/libros/secret/babini/Parcker\%20Part\%20Lucisano.pdf [Acceso: 09 de junio de 2020]

Paisley, W. (1984). Communication in the communication sciences. En Dervin y Voigt (eds). Progress in communications ciencies, vol, V, Ablex, Norwood, NJ, p 1-43.

Parra, M., Coutinho, R., \& Pessano, E. (2019). Um Breve Olhar sobre a Cienciometria: Origem, Evolução, Tendências e sua Contribuição para o Ensino de Ciências. Revista Contexto \& Educação, 34(107), 126-141. https://doi.org/10.21527/21791309.2019.107.126-141 [Acceso el: 10 de junio de 2020]

Pasquali, A. (1990). Comprender la comunicación (4ª ed.). Caracas, Venezuela: Monte Avila Latinoamericana.

Ramírez y Ramírez, K. M. (2009). Destellos de la comunicación. la diseminación de conocimiento a través de las publicaciones académicas (Digital). Razón y palabra Primera revista electrónica en América latina especializada en comunicación. $N^{o} 66$ (enero febrero). [online]. Disponible en: http://www.razonypalabra.org.mx/N/n66/varia/kramirez.pdf [Acceso: 09 de junio de 2020]

Ramírez, A. (2009). La teoría del conocimiento en investigación científica: una visión actual. Anales de la Facultad de Medicina, 70(3), 217-224. Disponible en: http://www.scielo.org.pe/scielo.php?script=sci arttext\&pid=S1025-55832009000300011\&lng=es\&tlng=es. [Acceso: 25 de mayo de 2020]

Ramos, C. (2001). Visión y revisión de las revistas académicas como parámetro de productividad científica: calidad, normas, políticas e indización. Extramuro, N ${ }^{\circ} 14$, p 27 62. Disponible en: http://190.169.94.12/ojs/index.php/rev_exm/article/view/11603 [Acceso: 09 de junio de 2020]

Requena.; J. (2003). ¿Cuánto cuesta hacer ciencia en Venezuela? Interciencia. Vol 28 (21-28) Disponible en: http://ve.scielo.org/scielo.php?script=sci arttext\&pid=S037818442003000100004\&lng=es\&tlng=es. [Acceso: 14 de mayo de 2020] 
Romero-Torres, M.; Acosta-Moreno, L.A.; Tejada-Gómez, M.A. (2013). Ranking de revistas científicas en Latinoamérica mediante el índice h: estudio de caso Colombia. Revista Española de Documentación Científica, 36 (1) Disponible en: http://redc.revistas.csic.es/index.php/redc/article/view/773/908 [Acceso: 14 de mayo de 2020]

Tapia, F. (2006) Quienes investigan e imparten docencia de calidad son los que mueven a la universidad. Visión Ucevista, 1, 4, 13-16.

Verardo, F. (2017). ¿Dónde están las mujeres? El techo de cristal y la carrera docente universitaria. Comunicación en el Seminário Internacional Fazendo Gênero 11 \& 13th Women's Worlds Congress (Anais Eletrônicos), Florianópolis, 2017, ISSN 2179-510X. Disponible en: http://www.en.wwc2017.eventos.dype.com.br/resources/anais/1499193319_ARQUIVO_DondeestanlasmujeresFlorenciaVerardo.pdf [Acceso el: 01 de junio de 2020]

Vincentelli, H. y G. Witter (2009) Producción científica: Revista de Pedagogía de la Universidad Central de Venezuela (1971-2005), Revista de Pedagogía, 30, 86, 161188. Disponible en: https://www.redalyc.org/pdf/659/65911663008.pdf. [Acceso: 15 de mayo de 2020]

Vincentelli, H (2003). Producción científica: Revista Paradigma (1990/1999) Paradigma, XXIV(1) pp. 177-186. Disponible en: http://revistaparadigma.online/ojs/index.php/paradigma/article/view/293 [Acceso: 15 de mayo de 2020]

Villegas, M.; González, F.; Bolívar, A. y Hernández, Y. (2005). La Producción Investigativa de la Jornada de Investigadores Junior de la UPEL Maracay. Paradigma Vol. XXVI (1) 241-280, Venezuela. Disponible en: http://revistaparadigma.online/ojs/index.php/paradigma/article/view/334 [Acceso: 15 de mayo de 2020]

Vessuri, H. (2006). La gobernabilidad de los riesgos de la ciencia y tecnología Interciencia. Revista de Ciencia y Tecnología de América, Vol. 31(4. 237. Disponible en: https://dialnet.unirioja.es/ejemplar/134110 [Acceso: 14 de mayo de 2020]

Witter, P. G. (1996). Avaliacão da producto científico sobre lectura na universidade. Psicología Escolar e educacional vol, 1, (1) p 31-38. Disponible en: https://www.scielo.br/scielo.php?pid=S1413-85571996000100005\&script=sci_abstract\&tlng=pt [Acceso: $14 \mathrm{de}$ mayo de 2020] 